\title{
Brain imaging tests for chronic pain: medical, legal and ethical issues and recommendations
}

\author{
Karen D. Davis ${ }^{1,2,3}$, Herta Flor ${ }^{4}$, Henry T. Greely ${ }^{5}$, Gian Domenico Iannetti ${ }^{6}$, \\ Sean Mackey ${ }^{7}$, Markus Ploner ${ }^{8}$, Amanda Pustilnik ${ }^{9}, 10$, Irene Tracey ${ }^{11}$, Rolf-Detlef Treede ${ }^{12}$ \\ and Tor D. Wager 13,14
}

Abstract | Chronic pain is the greatest source of disability globally and claims related to chronic pain feature in many insurance and medico-legal cases. Brain imaging (for example, functional MRI, PET, EEG and magnetoencephalography) is widely considered to have potential for diagnosis, prognostication, and prediction of treatment outcome in patients with chronic pain. In this Consensus Statement, a presidential task force of the International Association for the Study of Pain examines the capabilities of brain imaging in the diagnosis of chronic pain, and the ethical and legal implications of its use in this way. The task force emphasizes that the use of brain imaging in this context is in a discovery phase, but has the potential to increase our understanding of the neural underpinnings of chronic pain, inform the development of therapeutic agents, and predict treatment outcomes for use in personalized pain management. The task force proposes standards of evidence that must be satisfied before any brain imaging measure can be considered suitable for clinical or legal purposes. The admissibility of such evidence in legal cases also strongly depends on laws that vary between jurisdictions. For these reasons, the task force concludes that the use of brain imaging findings to support or dispute a claim of chronic pain - effectively as a pain lie detector - is not warranted, but that imaging should be used to further our understanding of the mechanisms underlying pain.

${ }^{1}$ Division of Brain, Imaging and Behaviour - Systems Neuroscience, Krembil Research Institute, Toronto Western Hospital, University Health Network, 399 Bathurst Street, Room MP12-306, Toronto, Ontario M5T 2S8, Canada. Correspondence to K.D.D. kdavis@uhnres.utoronto.ca
Great science brings great responsibility. In the field of pain research, developments in imaging technology are bringing closer the possibility of objective assessment of pain. In a clinical context, these developments could help physicians to understand and treat chronic pain. However, developments in imaging technology raise legal and ethical issues about the appropriateness of using such technology to assess chronic pain. The scientists who develop these technologies must take responsibility for their use beyond the scientific and clinical contexts.

Chronic pain is defined as pain that is present every day for $>3$ months (or is present on $\geq 50 \%$ of days for 6 months) or beyond the expected period of healing and does not have the warning function that acute pain does $^{1-4}$. Chronic pain is associated with enormous personal and societal costs; individuals with chronic pain often have a reduced quality of life and unmet therapeutic needs, and society is struggling to cope with the large numbers of people with this condition. Chronic pain affects up to $35 \%$ of the population, and costs of medical care and loss of wages and productivity are escalating $^{5-7}$. Efforts to improve prevention, treatment and rehabilitation for patients with chronic pain are ongoing. Chronic pain is also the subject of many legal disputes between patients, health care systems and disability benefit providers, in which proof or disproof that a patient is or is not experiencing pain might affect payments. Consequently, research to address whether chronic pain can be identified objectively is needed for a variety of reasons, particularly to provide evidence for insurance and legal purposes.

Given that the International Association on the Study of Pain (IASP) definition of pain is an "unpleasant sensory and emotional experience ${ }^{8,9}$ ", self-report, although subjective, is currently the gold standard for the assessment of pain. In drug development and clinical treatment, researchers and clinicians rely on self-reporting of pain (alongside other indicators of quality of life) to evaluate a patient's condition and the success of treatment. 
Neuroethics

A field that studies the implications of neuroscience for human self-understanding, ethics, and policy.

Arterial spin labelling An MRI-based brain imaging technique that detects cerebral blood flow based on magnetic tagging of blood.
Nevertheless, diverse groups - including patients, researchers, clinicians, pharmaceutical and medical device companies, insurers and the legal community seek methods for evaluating chronic pain besides selfreporting. Patients seek objective testing to demonstrate the reality of an invisible condition that is sometimes subject to doubt, researchers seek brain imaging markers that provide scientific, diagnostic and prognostic information that cannot be provided by patient self-reporting, and legal representatives and

\footnotetext{
Key points

- With suitable standardization and validation, brain imaging could provide objective biomarkers of key characteristics and mechanisms related to chronic pain, that could guide personalized pain management

- Brain imaging shows promise in the assessment of risk factors for chronic pain and in identification of the mechanisms that underlie transition to, and maintenance of, chronic pain

- Identification of brain-based markers of chronic pain requires technological advances, large-scale data acquisition across diverse groups of individuals, and strict application of standards of evidence

- Brain-based biomarkers should be used as an adjunct to rather than a replacement for subjective reports of the pain experience

- Use of brain imaging as legal evidence of an individual's pain is not advisable until the specificity and sensitivity of such tests are improved, and validated protocols exist

- Current brain-based measures should be used only to understand brain mechanisms underlying pain, factors that lead to persistence of pain, and targets in the brain for safe and effective pain management
}

officials seek techniques to supplement self-reporting and objectively support or challenge claims related to chronic pain.

Brain imaging technologies, including functional MRI (fMRI), PET, EEG and magnetoencephalography (MEG), have the potential to provide objective measurements of patterns of brain activity that underlie perceptual experiences (BOX 1). Consequently, some people are looking to brain imaging to provide a window into the experience of chronic pain, particularly because testimony based on fMRI was deemed to be admissible as evidence of pain in a 2015 state trial court in the USA ${ }^{10,11}$. This case was highly publicized, although the judgement was not published so no legal precedent was set, and the grounds on which the fMRI evidence was admitted were criticized by established experts in brain imaging studies of pain ${ }^{10,11}$.

In this context, the development of a brain imaging test for chronic pain ${ }^{12-14}$ has real-world consequences, so appropriate criteria for the use of such a test must be defined. Importantly, the way in which brain imaging evidence might be implemented in legal cases strongly depends on laws that vary greatly between countries. Therefore, examination of the capability of so-called 'pain-o-meter' brain imaging tests is critical, as is consideration of the use of such technology in light of societal views, neuroethics and legal issues. Thus, the aims of this Consensus Statement are to recommend criteria for the evaluation of neuroimaging measures of chronic pain, and to discuss the technical, biological, neuroethical and legal challenges related to establishing brain-based tests for chronic pain.

\section{Methods}

A presidential task force of the International Association for the Study of Pain (IASP) was established in 2015 to examine the feasibility of a brain-imaging-based diagnostic test for chronic pain. The task force had three purposes: to consider the capacity of brain imaging (in particular fMRI), on the basis of its technical and physiological constraints, to detect whether an individual has chronic pain; to place the capability of brain imaging as a diagnostic test for chronic pain in an ethical and legal context; and to establish guidelines for health care systems, governments and legal policy makers on the validity and ethics of adopting a brain-imaging-based test for pain. (BOX 2)

Members of the task force were chosen by the chair (Karen D. Davis) and IASP president to provide expert opinions across the fields of brain imaging technologies, basic and clinical science of pain, psychology, neuroethics, and the law. The members interacted via informal face-to-face meetings, email and conference calls to consider the scope of their task and the process by which the purpose could be achieved. Six small working groups were formed to discuss distinct topics (BOX 2).

Although the task force focused on standard blood oxygen level dependent (BOLD) fMRI, it did note that other imaging technologies, such as PET and arterial spin labelling (ASL), can measure cerebral blood flow in chronic pain, but are not yet widely available. The task force also noted that EEG has been used to assess 


\section{CONSENSUS STATEMENT}

\section{Box 1 | Sources of recommendations relating to brain decoding of mental processes}

Brain imaging techniques have greatly advanced our understanding of the neural basis of human perception, mental health and neurological disorders. Imaging has also opened the door to the possibility of brain decoding, a process in which information about human thoughts and feelings are derived from recorded brain activity. Several recommendations have been made by governments and academics on the use and misuse of brain decoding, including those listed here (links are included in the Further Information box):

- The US BRAIN (Brain Research through Advancing Innovative Neurotechnologies) Initiative

- The European Union Human Brain Project

- The Japanese Brain/MINDS Project

- The report of the ad hoc committee on Military and Intelligence Methodology for Emergent Neurophysiological and Cognitive/Neural Science Research in the Next Two Decades, created in 2004 by the US National Research Council at the request of the American Defence Intelligence Agency's Defence Warning Office ${ }^{123}$. A key recommendation (2.1) states: "the committee recommends further research on multimodal methodological approaches for detecting and measuring neurophysiological indicators of psychological states and intentions. This research should combine multiple measures and assessment technologies, such as imaging techniques and the recording of electrophysiological, biochemical, and pharmacological responses. Resources invested in further cognitive neuroscience research based on scientific principles and that avoid the inferential biases inherent in previous research in polygraphy."

- The 2011 UK Royal Society Brain Waves Project ${ }^{124}$ includes major discussions on "Neuroscience, society and policy" and "Neuroscience and the law".

stimulus-evoked abnormalities associated with neuropathic pain ${ }^{15}$ and is widely available, and is still being validated across populations for the assessment of different types of chronic pain.

To facilitate the working group discussions, the task force chair provided questions about acute and chronic pain, technologies, and ethical and medico-legal issues. Each working group developed a report that was circulated among members of the group for feedback and iterative modification. The reports were synthesized by the chair of the task force and circulated to all task force members for further discussion. The synthesized report underwent several rounds of modification as a result of the discussion among all task force members before it was finalized. The finalized report provided the basis for this Consensus Statement, which represents the culmination of the task force's findings.

\section{The science of pain and neuroimaging Distinctions in terminology}

In order to discuss the assessment of pain in any context, the meanings of commonly used terms must be clear. In clinical, scientific and legal settings, common misuses of pain-related terms include the inappropriate conflation of nociception and pain, and conflation of 'evoked' and 'ongoing' pain.

Pain versus nociception. The IASP defines pain as "an unpleasant sensory and emotional experience associated with actual or potential tissue damage, or described in terms of such damage" and nociception as the "neural process of encoding noxious stimuli ${ }^{8,9,16 "}$. On the basis of these definitions, therefore, pain is a complex, multifactorial and salient experience that comprises several elements. The perception that something hurts includes sensory-discriminative features (such as intensity, quality and location), cognitive-evaluative features, and affective-motivational aspects ${ }^{17}$. Moreover, chronic pain involves distress and disability, which often impairs functional activities in daily life ${ }^{1}$.
Pain and nociception are often related but are not equivalent, and can occur independently of each other. Given that pain is perceptual and exists only insofar as an individual experiences it, it can only be definitively identified through introspection and honest selfreporting. By contrast, nociception can occur without an individual being aware of it, and is not always dependent on consciousness; for example, measurable signs of nociception can be detected in people under anaesthesia, even though no conscious experience of pain is present ${ }^{18}$. fMRI and other brain imaging technologies measure indices of brain activity that can provide information about nociception and, by inference, pain, but brain imaging data can only be a proxy measure of pain. Consequently, any claims about an individual's subjective experience of pain that are based on decoded brain imaging and activity are necessarily inferential (as is the inference of pain on the basis of an individual's behaviour).

Evoked versus ongoing pain. Evoked pain can occur in an acute or chronic pain state and results from a stimulus. This stimulus can be either one that is normally painful, or one that is normally not painful (for example, the allodynia experienced in response to a light touch of the skin after a burn injury). Spontaneous or ongoing pain is pain that is detached from an overt external stimulus. A chronic pain condition can involve ongoing pain, evoked pain, or both types. The distinction between evoked and ongoing pain is important because their evaluation with brain imaging requires different acquisition parameters and approaches.

\section{Variability of pain}

The experience of pain varies tremendously within and between individuals, and this variability poses a challenge for the use of brain imaging findings as an objective biomarker of acute or chronic pain. Within an individual, the link between a noxious stimulus and the ensuing perception of pain is not direct; ascending nociceptive signals are modulated by 'top-down' control in 
the brain as well as by 'bottom-up' factors (such as inhibition by concurrent non-nociceptive input and other modulatory factors) that shape sensory input ${ }^{19}$ (FIG. 1). Furthermore, the relationship between the intensity of a noxious stimulus and the degree of pain is usually more direct in experimental studies, where the stimulus and the context can be controlled, than in a clinical context ${ }^{20}$. This observation highlights the difficulty in translating the results of laboratory studies of the nociceptive system to conclusions about the real-world experience of pain.

The experience of pain and the associated brain responses are also shaped by psychological factors, including learning and memory, personality traits and states, and cognitive, emotional, motivational, contextual and cultural variables ${ }^{21-23}$. Attentional focus and an expectation of pain or pain relief (for example, from a placebo) can also alter the experience of pain and the evoked brain activity ${ }^{21,23-30}$. Chronic pain can modify brain pathways involved in endogenous pain control ${ }^{21,22,25,31}$, making self-regulation of pain challenging. This form of brain plasticity varies between individuals with chronic pain, adds to the variability in pain processing, and might compromise treatment outcome.

\section{Imaging of pain}

Electrophysiological and imaging studies have shown that stimulus-evoked and acute pain is associated with activity in many brain areas that belong to different functional brain systems, rather than with activity in dedicated 'pain centres' within the brain (FIG. 1), including areas that are associated with coding information about noxious stimuli and reactions to noxious stimuli, the modulation of such information, the production of affective interpretations, attentional and emotional responses, and decisionmaking ${ }^{19,27,32-34}$. These brain areas include somatosensory, insular, cingulate and prefrontal cortices, and subcortical areas, including the amygdala, hippocampus, hypothalamus, ventral striatum, thalamus, periaqueductal grey, rostroventral medulla, numerous other brainstem areas and the cerebellum ${ }^{19,27,31,32,35-37}$. Neuronal oscillations in these areas differ from each other in frequency ${ }^{38}$, and dynamic and flexibly accessible brain networks are formed between these regions that subserve multiple functions.
Importantly, the fact that engagement of these brain areas has been associated with the perception of pain does not imply that the observed cortical activity is necessary for the perception of pain; dissociation of activity in these brain areas and the intensity of nociceptive stimuli and perceived pain has been demonstrated in several studies ${ }^{39-43}$.

The original 'body-self neuromatrix' concept ${ }^{44,45}$ led to use of the term 'pain matrix' to describe the brain regions involved in the pain experience. However, this term has been superseded for several reasons, including the fact that it referred to a restricted set of brain regions, and that activity of these regions is nonspecific to pain ${ }^{41,46-48}$. The assumption that an experience of pain can be inferred by observing activity in parts of this ill-defined set of regions has led to much confusion about the importance of neuronal activity in these regions in relation to pain $^{49}$. Nevertheless, this inference served as a starting point for the development of machine learning and related decoding techniques to identify more-precisely defined patterns of activity.

Beyond regional activity, acute and chronic pain engages intrinsic brain networks (also known as resting state networks) - such as the default mode, salience and somatosensory networks, which together subserve homeostatic, attentional, cognitive, emotional, executive, and sensory functions $s^{50}$ - and alter their functional connectivity. No single definition of the boundaries of these networks exists, but the salience and somatosensory networks as defined in large-scale resting-state studies overlap extensively with the regions that were typically included in the pain matrix. The combination of these intrinsic networks and the top-down control systems that are engaged during the experience of pain across moment-by-moment and long-term timeframes has been termed the dynamic pain connectome ${ }^{50}$. However, the observation that activity within the dynamic pain connectome occurs when a person is experiencing pain is not sufficient to conclude that such activity is an indicator of pain, or that it is related to pain at all. To make this conclusion, the activity must relate to reporting of pain, and must not occur in the absence of pain.
Body-self neuromatrix

A theory proposed by Ronald Melzack, in which a set of brain regions is considered to be a flexible reference space for the integration of many diverse processes into a representation of pain-in-context.

\section{Box 2 | Purposes and working groups of the International Association for the Study of Pain task force}

\section{Purposes}

- To consider the capabilities of brain imaging (particularly functional MRI) to detect whether an individual has chronic pain

- To place the capability of brain imaging as a diagnostic test for chronic pain in an ethical and legal context

- To establish guidelines for health care systems, governments and legal policy makers on the validity and ethics of adopting brain-imaging-based testing for pain

\section{Working groups}

- Acute and chronic pain and nociception: behaviour and neurophysiology (Chair: Gian Domenico lannetti)

- Acute and chronic pain psychology (Chair: Herta Flor)

- Brain imaging technologies (Chair: Tor D. Wager)

- Brain imaging of acute and chronic pains (Chair: Markus Ploner)

- Proof of pain and veracity in legal cases involving pain (Chair: Amanda Pustilnik)

- Neuroethics and the law (Chair: Amanda Pustilnik) 


\section{CONSENSUS STATEMENT}

a
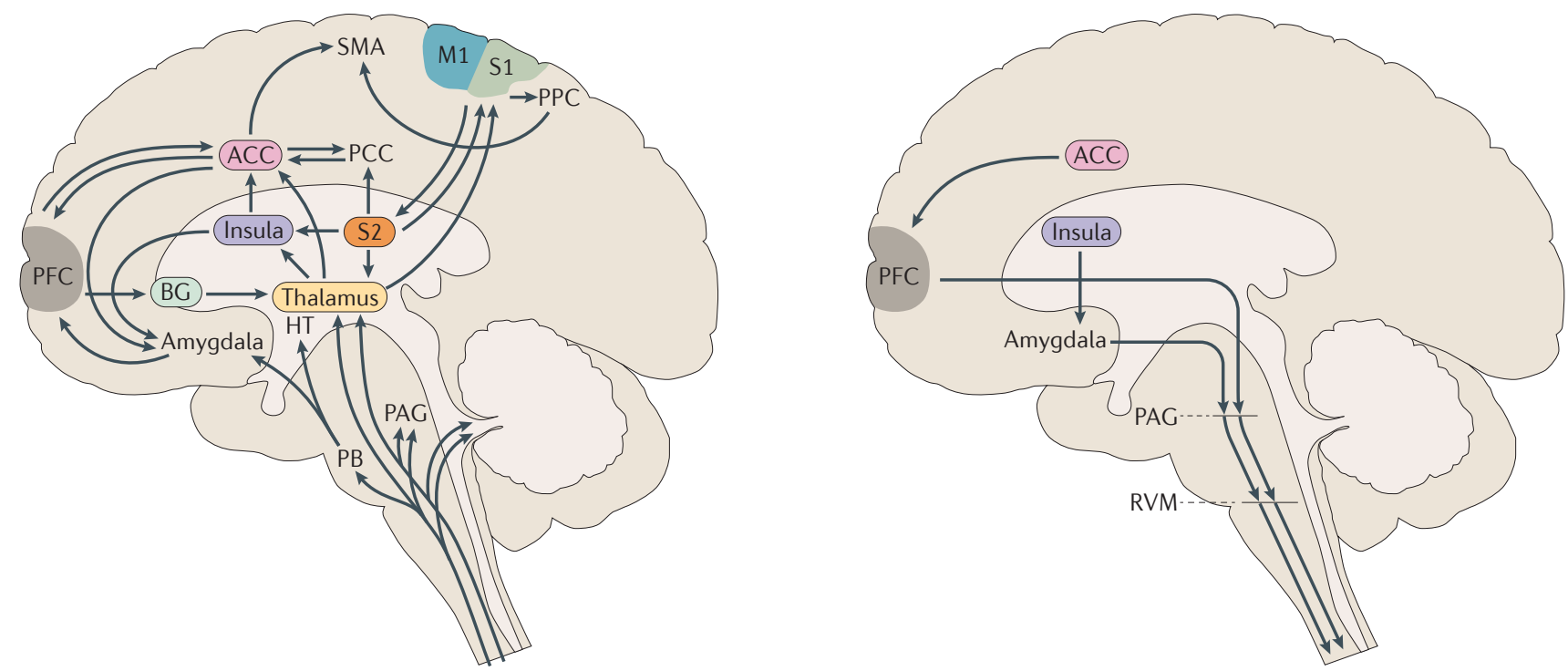

b Mid cingulate cortex

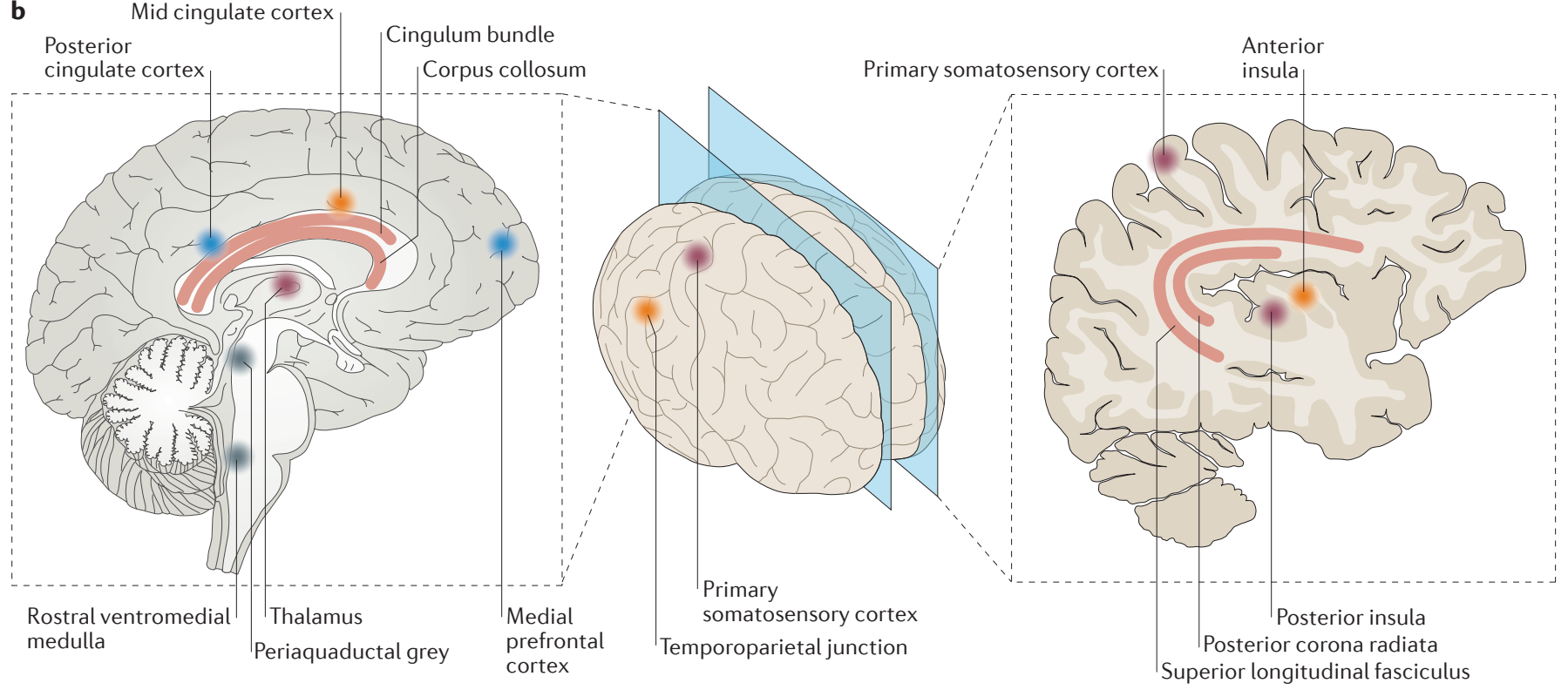

Default mode network

Salience network

Sensorimotor network

White matter bundles

Figure 1 | Brain pathways, regions and networks involved in acute and chronic pain. a | Major ascending (bottom-up) pathways from the spinal cord to the brain that are activated by noxious stimuli (left) and descending (top-down) pathways that modulate transmission of ascending nociceptive signals (right). $\mathbf{b} \mid$ Key brain areas, resting state functional networks and white matter in which activity is often found to be abnormal in chronic pain. Permission for part a left panel obtained from John Wiley and Sons (C) Apkarian, A. V. et al. Eur.J. Pain 9, 463-484 (2005). Permission for part a right panel obtained from the American Society for Clinical Investigation @ Schweinhardt, P. \& Bushnell, C. J. Clin. Invest. 120, 3788-3797 (2010).

\section{Decoding pain from brain activity}

Nociceptive stimuli trigger a broad variety of cognitive, emotional, motivational, autonomic and motor processes that are not specific to pain but are part of the multidimensional nature of the pain experience. Consequently, many (if not all) features of brain activity that have been associated with pain are not specific to pain. As a result, making inferences about whether a person is experiencing pain on the basis of current brain imaging techniques is problematic. Nevertheless, efforts are being made to decode pain from brain activity ${ }^{51-54}$. Similar efforts are being made to predict pain from brain structure, such as grey matter volume and white matter connectivity, but these efforts were beyond the scope of this task force and are discussed elsewhere ${ }^{55-58}$.

Univariate and multivariate approaches have been applied to the analysis of brain imaging data in order to decode pain. In univariate approaches, brain metrics 


\section{Box 3 | Defining a putative biomarker of acute experimental pain}

\section{Imaging technique}

- fMRI: (evoked, resting state connectivity), quantitative cerebral perfusion imaging

- PET: rCBF (water), glucose metabolism, receptor binding (for example, serotonin, opioid, dopamine)

- EEG, MEG

\section{Activation features}

- Presence or absence of an evoked response

- Magnitude, sign (increase or decrease from baseline)

- Polarity, amplitude and peak latency of evoked potentials

- Scalp distribution (EEG or MEG)

- Anatomical location (fMRI or PET) of the evoked response

- Size and spatial extent (fMRI or PET activations)

- Connectivity with other brain areas

- Behavioural correlation (for example, pain intensity)

\section{Data acquisition parameters}

- MRI field strength (for example, 1.5 T, 3.0 T)

- Pulse sequence (for example, echoplanar, spiral)

- Spatial and temporal resolution

- Amplifier common mode rejection

- Number and position of EEG electrodes and reference site

- EEG amplification and filtering

- Trial averaging

Protocol design

- Stimulus modality (heat, mechanical, electrical, laser)

- Stimulus intensity (fixed stimulus, fixed evoked pain intensity)

- Control condition (no stimulus, nonpainful (for example, thermal or mechanical) stimulus)

- Duration and number of trials (block versus single trial design)

- Preprocessing and statistical criteria

- Haemodynamic response (stimulus-related versus percept-related)

- Spatial (Gaussian) and temporal filters

- Height and extent (cluster) threshold

- Correction for multiple comparisons

-Whole brain versus region of interest analysis

- Fixed effects versus random effects analysis

\section{Physiological issues}

- Brain areas are nonspecific and multi-responsive (fear, attention, salience, emotion, pain)

- Overlap of pain-responsive and pain-nonresponsive neurons for touch (no brain area yet to be found to contain only pain-responsive neurons)

- BOLD, rCBF ceiling effects

Biomarkers should be specified in comparison to a reference database defining the effect of demographics such as age, sex and health status (for example, comorbid vascular disorders). BOLD, blood oxygen level dependent; fMRI, functional MRI; MEG, magnetoencephalography; rCBF, regional cerebral blood flow. Permission granted by Wolters Kluwer. Please contact the publisher for reuse terms and conditions.
(BOX 3). Multivariate approaches integrate multiple features of brain imaging data into an integrated predictive model $^{3,4,59,60}$. Machine learning and statistical techniques are often used to identify patterns in these data, and are optimized to jointly predict patient status, the experience of pain, analgesia, and other outcomes. These approaches have been successfully used to decode some aspects of stimulus-evoked acute pain from patterns of brain activity, at least to some extent ${ }^{51,61-71}$ (BOX 4).

Interpretation of these analyses, however, requires caution owing to several important limitations. First, successful detection of pain does not imply that the experience of pain is what the predictive brain signature measures. Machine learning might lock onto features that correlate with pain, such as salience, rather than pain itself - the reverse inference problem ${ }^{72,73}$ (discussed further below). Second, successful prediction of pain does not imply that the predictive brain biomarker is specific to the experience of pain; such a neuromarker must be tested in many types of painful and non-painful conditions to empirically establish what it does and does not respond to ${ }^{54,74-76}$. Third, an imaging neuromarker might not generalize to all types of pain, or to all individuals; this aspect must also be tested empirically. Fourth, prediction of pain does not imply a causal relationship between the predictive activity and the experience of pain.

Most work to date has involved attempts to identify neuromarkers that reflect mechanisms and neurophysiological processes that are important in chronic pain $^{31,64,77-81}$. Given that the experience of pain has diverse influences, from nociception to social context, it is very unlikely that a single neuromarker will be found to reflect all aspects of acute and chronic pain in all contexts.

\section{Assessment of chronic pain}

Chronic pain is currently assessed by medical history, clinical examination, questionnaires, behavioural measures, and, occasionally, laboratory tests. The key symptom of most types of chronic pain is stimulusindependent pain. Such spontaneous, ongoing pain can be steady or might fluctuate over time in several patterns. In some conditions, chronic pain is associated with hypersensitivity to sensory stimuli and/or a decoupling of nociceptive stimuli from painful percepts (altered evoked pain). Chronic pain can occur in the absence of tissue damage in the painful region (for example, referred pain or neuropathic pain) or an identifiable lesion, and even without any peripheral input, as in central neuropathic pain (for example, pain associated with spinal cord injury and post-stroke pain). The neuropathology that changes electrical, neuroimmune and/or neurochemical signalling might not be detectable with current noninvasive techniques. Chronic pain often co-occurs with a broad variety of emotional, cognitive and motivational changes, including mental disorders, which complicates the identification of a specific neuromarker of chronic pain.

Imaging of chronic ongoing pain requires a different approach to that used for imaging of acute, experimentally evoked pain. Chronic ongoing pain can remain constant or vary slowly during the course of a brain imaging 
Box 4 | Brain decoding of acute experimental pain with multivariate pattern analysis and machine learning

Machine learning is an analysis approach that exploits the ability of computers to learn from, and make predictions from, different kinds of data ${ }^{125}$. When applied to functional brain images, machine learning can be used to detect response patterns (for example, intensity and spatial distribution of functional MRI (fMRI) signals or spatial-temporal patterns of EEG signals) associated with a given experimental variable (for example, the intensity of pain perception) ${ }^{61,126}$.

Multivariate pattern analysis (MVPA) is a machine learning technique in which a pattern classifier identifies the $\mathrm{fMRI}$ responses elicited by different stimuli ${ }^{3,4,59,60}$. MVPA is different from conventional univariate $\mathrm{FMRI}$ analysis approaches, which use a general linear model to detect average regional activity and consider a single voxel at a time within a given brain region (see figure, top right). MVPA detects patterns of activity across many voxels, to determine whether (and how) a condition is represented in the spatial pattern of activity sampled across the multiple voxels of a given brain region (see figure, bottom right). Consequently, MVPA is usually more sensitive than conventional univariate analysis to fine, within-region spatial differences in brain activity across experimental conditions, and can detect changes in the spatial distribution of fMRI signals even when average regional activity does not differ across different conditions. The patterns of activity identified by MVPA can be used to predict the occurrence of different experimental variables (for example, different levels of subjective pain, or pain versus touch). The validity issues raised by the potential for reverse inference also apply to the interpretation of the results obtained using machine learning. However, a given machine-learning result can be interpreted to reflect a pain signature only if the relationship between the brain response pattern and pain is unique for pain ${ }^{8}$.

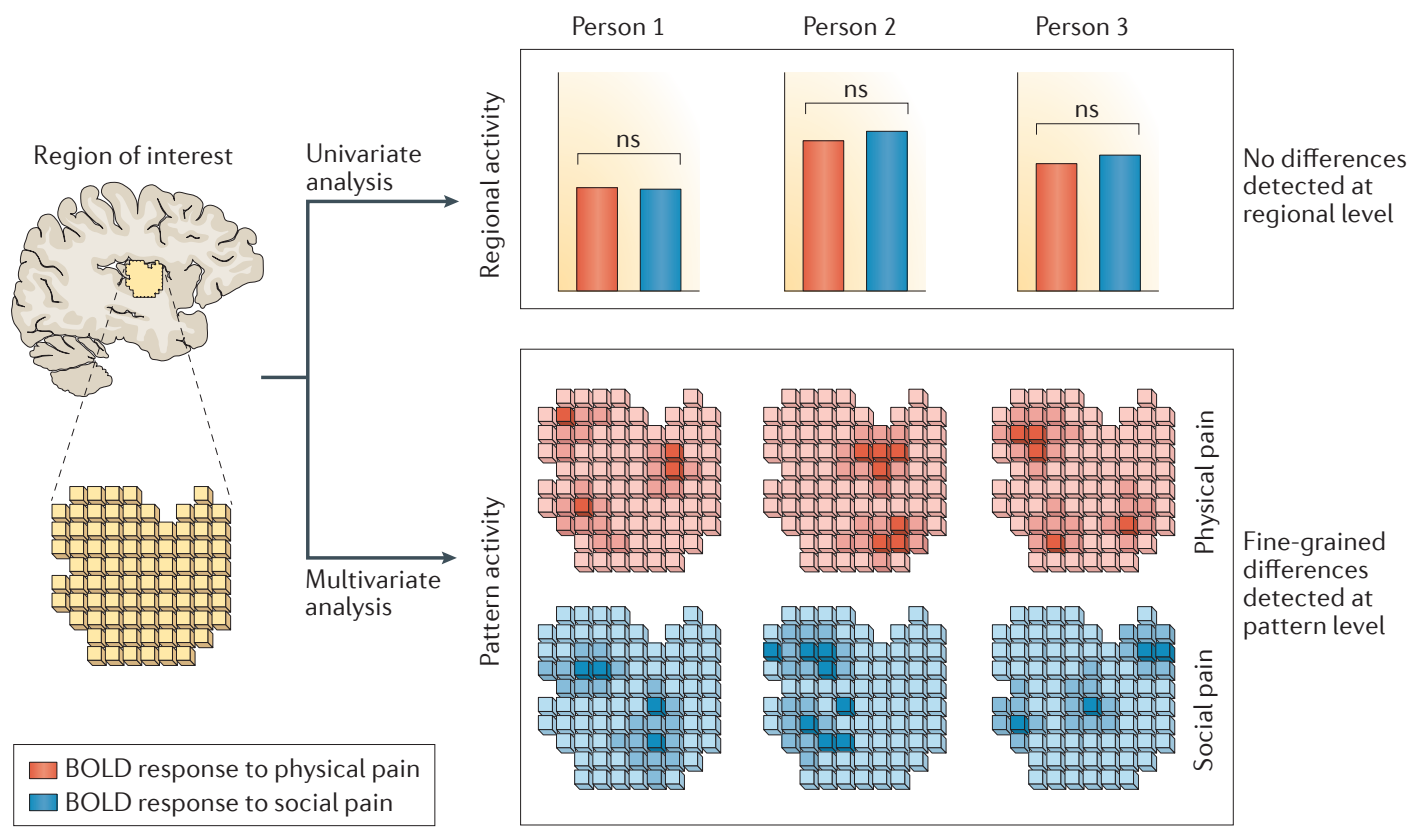

Modified with permission from Elsevier @ lannetti, G.D. et al. Trends Cogn. Sci. 17, 371-378 (2013). Contact Elsevier for reuse terms and conditions.

session, rendering it essentially invisible to traditional imaging techniques, such as stimulus-evoked fMRI. Furthermore, chronic pain involves qualities of experience, and possibly other dysregulated brain processes, that are not always characteristic of acute experimental pain in healthy people. Therefore, we cannot assume and it is highly unlikely - that chronic pain generates precisely the same brain features as those observed in acute experimental pain.

In principle, functional brain imaging can measure three types of activity relevant to chronic pain: evoked activity, task-free resting-state brain activity, and activity related to a particular attribute of ongoing clinical

\section{Hyperalgesia}

Greater pain than is normally evoked by a specific noxious stimulus.
Evoked activity. In some conditions, chronic pain is accompanied by hyperalgesia and/or allodynia, which are signs of peripheral and/or central sensitization. This sensitization can be studied by applying brief stimuli, preferably with multiple stimulus intensities to characterize stimulus-response functions ${ }^{82,83}$. With this approach, one can determine the stimulus-evoked brain responses that differ between patients with chronic pain and healthy individuals, or between responses elicited by stimuli applied to affected and unaffected areas of the same patient ${ }^{83-86}$. Alternatively, brain responses that correlate with pain intensity ${ }^{79,82,87-91}$, or perceptrelated brain activity that fluctuates in synchrony with the experience of pain ${ }^{31,69,89,92-96}$ can be identified. Association of activity with the experience of pain is particularly important in chronic pain, because an evoked pain response can be out of sync with or completely disconnected from the timing and duration of the applied stimulus ${ }^{89,20,97,98}$. 
Resting-state brain activity. Resting-state, or 'task-free', brain activity is widely assessed in many types of disorders. Resting-state fMRI involves the acquisition of fMRI data in the absence of any overt stimulus or task. The data can be used to measure functional brain connectivity that relates to a combination of spontaneous thought processes and ongoing neural and physiological maintenance processes; in chronic pain, these processes include those involved in ongoing pain ${ }^{50}$. Moreover, variability in brain activity can provide insight into brain health, pain sensitivity and the capacity for brain plasticity ${ }^{99}$; these measures can differ between patients and healthy individuals, or vary in relation to characteristics of pain and risk factors for pain or its chronification $^{26,31,100-103}$, so can be clinically useful. A challenge in this approach is that the nature of any particular change in the pattern of resting-state connectivity associated with pain has not been determined, as patterns are altered in a range of clinical conditions; this uncertainty makes it unclear whether any particular pattern is related to pain itself, spontaneous thought, or other related processes.

Ongoing clinical pain. Patients with chronic pain can experience ongoing pain, often with no obvious external cause. This type of pain can change over time, and these dynamics can engage brain areas and networks associated with emotional, cognitive and motivational processes ${ }^{50,104}$. However, brain measures associated with ongoing pain can be quite different from those that correlate with evoked pain ${ }^{64}$.

PET-based or ASL-based measurements of cerebral blood flow ${ }^{105}$ can be used to examine ongoing clinical pain, because these techniques are quantitative and do not require delivery of an external stimulus. Early ASL studies were limited by relatively weak blood flow measurement signals, which typically needed to be boosted by manipulations that increase pain ${ }^{106}$, but modern ASL methods, scanners and analyses, along with improved experimental paradigms, compensate for this limitation. A different approach is to measure the dynamic brain connectivity, which varies over time, to enable identification of connectivity patterns that vary with reported levels of ongoing pain. This approach has revealed changes in the default mode and salience brain networks in chronic pain states ${ }^{107-109}$. However, a potential confounding factor is the possibility that the judgement and reporting of ongoing pain causes a detectable change in brain processing.

\section{Challenges in imaging chronic pain}

Imaging of pain-related processes in patients is challenging owing to special considerations. These challenges include variability in imaging within and between patients, the specificity of the imaging findings, the possibility of reverse inference, and various technical and statistical issues.

Patient variability. Good consistency across hundreds of brain imaging studies of healthy people has enabled identification of a core set of brain areas that respond to acute noxious stimuli ${ }^{31,35}$ at a group level. At the individual level, however, specific patterns and levels of evoked activation vary ${ }^{31,35}$. Similarly, dozens of imaging studies across various chronic pain conditions have identified some general abnormalities in patients compared with age-matched and sex-matched healthy controls, but these findings vary at the individual level ${ }^{31,35}$. Furthermore, identification of consistent pain-related activation or connectivity in the brains of individuals is challenging not only because of differences between healthy people and between patients, but also because data obtained from a single individual have low statistical power. Moreover, each moment in time in each person is marked by a unique contribution of sensory, cognitive, emotional and motivational processes (as well as physiological and demographic states) to the experience of pain. Consequently, the brain activity associated with acute and chronic pain varies across time, people and context.

Multivariate, machine-learning-based measures have identified a core pain-related network that enabled tracking of within-person variations in pain in a restricted cohort ${ }^{61,63,80}$. This finding is encouraging, but the model is incomplete because these measures do not capture the entire pain experience (for example, qualities and emotions) and do not account for changes in attention or salience. In addition, between-person variables that affect the fMRI signal (for example, caffeine, haematocrit levels and changes in the neurovascular system associated with ageing and disease) and brain processes involved in making verbal reports or decisions diminish the ability to accurately predict which individuals experience more pain than others. Consequently, no normative database of acute pain responses or functional connectivity across age, sex, ethnicity and other relevant variables exists to enable comparisons with an individual who presents with chronic pain.

Specificity. No brain areas or networks have yet been specifically and exclusively linked to chronic pain. Furthermore, many processes that are associated with chronic pain can also occur in many other states. For example, many abnormalities observed in chronic pain conditions are also present in depression, anxiety and other conditions ${ }^{21,27}$. This combination of substantial overlap of chronic pain with other processes that are not necessarily pain-related, and comorbidity with mental disorders, implies that an inherent lack of specificity is a fundamental problem for brain imaging-based diagnostic tests for chronic pain.

Reverse inference. The reverse inference problem is a central obstacle in accurate brain decoding to determine whether an individual is or is not experiencing chronic pain. In this context, reverse inference means the inference of a particular mental state (for example, the perception of pain) from a given pattern of brain activation. The likelihood of such an inference being correct depends on the degree to which activation in that pattern occurs exclusively as a result of pain ${ }^{54,110,111}$. Accurate assessment of whether a reverse inference is true requires not only assessment of how often the 


\section{Box 5 | Proposed criteria for establishing valid neuroimaging protocols}

- Criterion 1: A precise definition of a pain neuromarker

- Criterion 2: Applicability of the pain neuromarker to individuals

- Criterion 3: Methodological procedures used during testing must be validated

- Criterion 4: Measures must be internally consistent and image data quality validated for the individual tested using positive and negative controls

- Criterion 5: The neuromarker must be diagnostic for pain

- Criterion 6: The neuromarker must be validated with converging methods

- Criterion 7: The neuromarker must be generalizable to the patient group tested and to the test conditions

A given brain measure could meet these standards with varying degrees of rigour, and different levels of evidence might be appropriate for different clinical and legal applications. In addition to the scientific evidence, applications of brain markers for nociception and pain should also take into account social implications (including financial, social and other costs), and the effects of errors in both false-positive and false-negative directions. effect

Limiting of the magnitude of fMRI responses owing to the upper limit of blood flow; the effect can also be related to nonlinearities in vascular responses that are in turn related to stimulus history effects.
Haemodynamic ceiling

pattern of brain activity occurs when pain is experienced, but also how often the pattern is present when pain is not experienced. Furthermore, many processes associated with chronic pain are not pain-specific, so determining how often the network is activated when pain is present is not enough: one must also demonstrate that the same network is not activated when no pain is present. The inference that network activity indicates pain cannot be assumed, but must be demonstrated with data that are sufficient to establish specificity and sensitivity. Importantly, some brain imaging responses associated with pain have also been observed in response to equally salient but not painful stimuli ${ }^{71}$. This observation indicates that an inference of causality

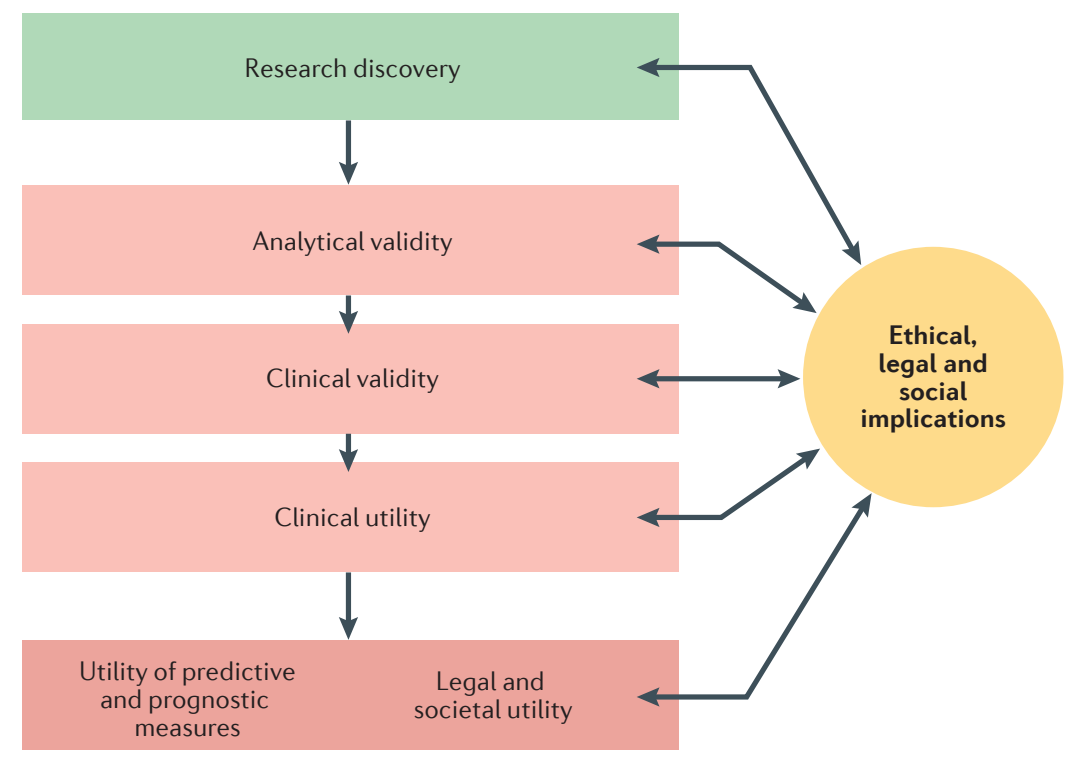

Figure 2 | The US Centers for Disease Control and Prevention Office of Public Health Genomics ACCE Model Project. The ACCE (analytic validity, clinical validity, clinical utility and associated ethical, legal and social implications) model is a framework of four key factors set out by the Centers for Disease Control and Prevention Office of Public Health Genomics to evaluate new genetic tests for validity and utility. This model can also be applied to the field of neuroimaging-based biomarkers of pain, which is currently in a research discovery phase.
- that the experience of pain is a direct result of the network activity detected with brain imaging - might be incorrect. Demonstrating that a given fMRI response pattern (or indeed any pain biomarker) has a high sensitivity and specificity as a test does not necessarily imply that the neural activity from which that pain biomarker is derived corresponds to the neural activity through which pain emerges as a percept.

Technical and statistical challenges. The outcome of any brain imaging session is highly dependent on many study design variables, data acquisition imaging parameters, approaches to data pre-processing and statistical analysis, and the statistical significance level used. Currently, neither a gold-standard pre-processing pipeline for detecting chronic pain nor standards for procedures and quality control have been established ${ }^{112}$ (BOX 3). Moreover, the relationship between fMRI signals, neurovascular coupling and neuronal activity is not fully understood ${ }^{113}$. Furthermore, some inherent assumptions about the haemodynamic response and vascular reactivity might be violated for some people, owing to age, obesity, stroke and other neurovascular complications ${ }^{114-116}$. The haemodynamic ceiling effect and baseline blood flow both affect the magnitude of stimulus-evoked fMRI responses, and should be considered when using fMRI to assess the magnitude of evoked responses and analyse connectivity. For example, if ongoing pain is associated with high levels of activity in nociceptive neurons and high levels of tonic blood flow to the areas of the brain that contain those neurons, the additional neuronal activity associated with evoked pain might be undetectable on $\mathrm{fMRI}^{117}$. These factors make paramount the inclusion of control tasks (which are often included in pharmacological imaging studies but rarely in other types of imaging studies) to enable assessment of such influences.

\section{Criteria for neuroimaging protocols}

In this section, we present criteria for the evaluation of fMRI measures of pain; these criteria also apply to any other biomarker of pain (BOX 5). A given brain measure might meet these criteria with varying degrees of rigour, and different levels of evidence might be appropriate in different clinical and legal contexts. Many of these criteria are consistent with the standards set out by the US Centers for Disease Control and Prevention Office of Public Health Genomics ACCE (Analytic validity, clinical validity, clinical utility and associated ethical, legal and social implications) Model Project, the aim of which was to devise standards of evidence for use in genetic testing for disorders (FIG. 2). The establishment of similar benchmarks and standards for neuroimaging studies to assess chronic pain and to determine the validity of test results would provide an equivalent resource.

We note that the criteria we propose are not yet adequately fulfilled - the goal of this Consensus Statement is to provide a framework for the development of valid protocols for neuroimaging of chronic pain. We therefore encourage individual and collective efforts, including collaborative multisite projects and data-sharing 


\section{Box 6 | Potential uses and misuses of pain diagnostics based on brain imaging}

\section{Potential uses (pros)}

- Improve pain treatment

- Optimize intervention and/or treatment

- Assess potential adverse effects of treatment on the basis of brain effects

- Confirm eligibility for insurance and coverage for specific treatments

- Pain diagnostics for non-communicative populations

- Personalized medicine

Tailored treatment based on individual responses and capabilities

Potential misuses (cons)

- Consequences of false-negative findings

- Doctor-patient, employee-patient, family-patient trust issues

- Denial of medical treatment or insurance coverage

- Mental health, stress, spousal and/or family issues

- Financial, insurance and employment issues

Privacy and legal (for example, medical malpractice) issues

- Consequences of false-positive findings

- Unnecessary, costly and potentially harmful analgesic treatment in non-communicative patients

- Human, infrastructure, financial and time resources

- Misunderstanding as a substitute for self-report

Permission granted by Wolters Kluwer. Please contact the publisher for reuse terms and conditions.

\section{Criterion 3}

Methodological procedures used during testing must be validated. The methodological procedures used to test for a neuromarker (BOX 3) in an individual must be clearly defined and validated. These procedures include methods for instructing the patient, assessing and minimizing confounding factors (for example, head movement and physiological artefacts), data acquisition, data processing and analysis, application of the neuromarker (how to calculate the neuromarker's response value or score from the neuroimaging data obtained), and interpretation of the results.

\section{Criterion 4}

Measures must be internally consistent, and the quality of imaging data validated for the individual tested, using positive and negative controls. Testing for pain neuromarkers requires complex data acquisition and analysis steps that are subject to artefacts, physiological noise and technical problems. Defined procedures must be established for determining at the time of the test whether the quality of the data and their analysis are adequate for detection of signals in a particular individual. Demonstrating the neuromarker's internal consistency the ability to consistently obtain a valid, reproducible, and reliable measure - is critical. Positive controls must also be established to enable validation in the individual being tested. Such controls would be patterns of brain activity that are independent of pain and that must be observed for the test to be considered valid. Similarly, negative controls must be established - these controls would be patterns of brain activity that must be absent for the test to be considered valid. In addition, controls must be in place to enable detection of feigned responses or deception, such as responses to deliberately self-inflicted pain.

\section{Criterion 1}

Precise definition of a pain neuromarker. Any brain measure that is to be used as a neuromarker of pain must be precisely defined, and procedures must be established for determining whether the data are of suitable quality for a valid test, calculating the measure from an individual's imaging data, and determining appropriate cut-off values for the measure. Definitions must go beyond a simple description of activity in a brain area; they should specify the precise voxels of interest within the involved brain region or regions, the relative activity across voxels, the voxels that should be included for the individual (if the set of voxels used is individualized according to sex, age or other factors), and the relative expected magnitude of activity across voxels. The current standard brain mapping procedures are usually not defined precisely enough to produce useful neuromarkers.

\section{Criterion 2}

Applicability of the pain neuromarker to individuals. For a neuromarker of pain to be useful, its accuracy in individual people must be established (see Criterion 5). In addition, the ability to apply a neuromarker to an individual's brain imaging data without prior knowledge of that individual's pain must be demonstrated.

\section{Criterion 5}

The neuromarker must be diagnostic for pain. The accuracy of a neuromarker for detecting and/or quantifying pain must be established, including quantification of its sensitivity, specificity, positive predictive value and negative predictive value for pain at the level of the individual person. Determining these metrics requires knowledge of, or assumptions about, the true pain levels in a validation sample (for example, the true pain intensity in a group of individuals who have chronic pain and a group who do not have chronic pain). In addition, calculating the positive and negative predictive value of the measure requires knowledge about the prevalence of the chronic pain condition of interest in the general population. Some neuroimaging data that have previously been submitted to court in legal cases did not include sensitivity and specificity values, as these metrics were never assessed ${ }^{10}$. Such tests have been admitted as evidence in court, but we would argue that they should not be deemed admissible without an established predictive value.

The neuromarker must be validated with converging methods. An imaging neuromarker of pain is more likely to be valid if its relationship with pathophysiological

\section{Criterion 6}


mechanisms of chronic pain has been established by comparison with other methods (for example, invasive human or animal studies). Regardless of the brain systems involved, a neuromarker has increased plausibility as a measure of pain when a strong relationship has been demonstrated between the neuromarker and pain in different populations studied with different methods.

\section{Criterion 7}

The neuromarker must be generalizable to the patient group tested and to the test conditions. The clinical diagnostic value of a neuromarker (for example, its positive and negative predictive values) might be different for different populations, and a predictive value estimated in one population (for example, healthy individuals) cannot be assumed to apply in another population (for example, patients with back pain), unless the marker can be shown to generalize across populations, or good evidence supports the assumption that it generalizes. The test must also be validated under the conditions to be used in the applied test; these conditions include elements such as the neuroimaging equipment (for example, the specific scanner), the analysis software and procedure, the psychological and physiological condition of the person (for example, their prior sleep and food and drug intake). A marker that is validated with one acquisition and analysis procedure is not necessarily valid if applied under other procedures, unless evidence shows that the marker is generalizable across variations in scanners and procedures. Given that fMRI measures signals relate to the regulation of brain perfusion and oxygen consumption, the presence of a neurovascular disease might change fMRI neuromarker values independently of neural activity. Such sources of variation must be considered and controlled for (when possible) to enable appropriate interpretation of the signal.

\section{Legal and ethical issues}

\section{Chronic pain and the legal system}

Chronic pain is an issue in a broad range of legal matters, accounting for tens of thousands of legal cases and billions of dollars of civil damages and benefit payments annually. Legal decisions involving claims of chronic pain might include determinations of eligibility for disability or workers' compensation benefits, tort claims, and entitlement to public or private insurance ${ }^{14}$. Assessment of chronic pain can also affect the extent to which a person is protected by disability law.

A survey of legal cases in the USA, Canada, and UK shows that people whose claim of chronic pain is supported by strong evidence might nonetheless be denied benefits, compensation or disability status ${ }^{118}$, while people with actually or potentially fabricated claims of chronic pain might receive compensation, because legal decision-makers apply variable and unreliable standards to these cases ${ }^{14,119}$. Rigorously validated and regulated brain imaging techniques for assessing pain might, therefore, have the potential to improve legal practice and increase average accuracy and fairness across health-care-related cases. Besides this potential future role of brain-imaging-based evidence of pain in an individual, our current understanding of the science of chronic pain (which is grounded in brain imaging studies) can inform legal decision-makers about the general features of how chronic pain occurs, presents and endures. This body of research can guide the crafting of more accurate and precise laws that relate to pain as a source of disability, and can assist the evaluation of evidence in individual cases.

Whether a brain imaging test is used to support or refute a claim of pain, it must meet rigorous standards. First, the test must meet a high standard of evidence, satisfying all of the scientific criteria proposed above, as well as the legal criteria of the relevant jurisdiction. Second, and more important, the test must recognize that each individual is unique, and that abnormal brain activity or structure alone does not prove that an individual is experiencing pain. Individual differences that result from factors such as genetics, environment and prior learning can create difficulties when comparing individual scans to group-averaged findings. Accordingly, brain imaging tests that are sufficiently rigorous to meet scientific and legal criteria should not be considered to stand alone, but instead should be assessed in the context of an individual's current medical and behavioural profile and his or her prior experiences. For a neuromarker to be believable, therefore, one would need to be able to evaluate the extent and effect of neuroplasticity, brain reorganization, or other individual differences, as noted above. In principle, such individual differences could never be entirely ruled out, although this difficulty does not preclude such evidence from being useful. In this context, legal officials would have to assess the strength of the correlation, as is the case with other scientific evidence: even DNA identification never can provide certainty.

\section{Chronic pain assessment in legal cases}

Brain-based evidence of chronic pain could be used in legal matters for two distinct purposes. The first is to educate decision-makers about the pathophysiology of chronic pain as a foundation on which to assess the evidence pertaining to a specific case. As an educational tool, brain imaging can be used to explain chronic pain conditions, but admission of such evidence should be accompanied by a caution that the evidence provides background information that can only be used to assess the facts of a claim, and does not prove or disprove any individual claim. Furthermore, some research suggests that jurors and other decision-makers can be unduly influenced by being shown visually appealing brain images ${ }^{120}$; for this reason, courts should consider whether to limit expert evidence on the neurobiology of chronic pain to verbal testimony. The second purpose of brain-based evidence in legal matters is to attempt to provide evidence for or against the presence of pain and its intensity in a particular individual. To date, researchers have not addressed this issue, but have instead addressed fundamental questions about the representation of pain in the brain, mechanisms relevant to the development, exacerbation and maintenance of persistent and chronic pain, and how to identify targets 
for treatment. On the basis of what is currently known, brain imaging is not sufficiently reliable to be used as a pain detector to either support or contradict an individual's self-report.

\section{Future of brain imaging in legal cases}

In the future, brain imaging of pain might enable assessment of a person's sensitivity to evoked pain or to pain treatment, and possibly of a person's susceptibility to developing chronic pain ${ }^{14,121}$ (BOX 6). Such information could be used for many purposes: to determine the likelihood that a person with chronic pain will recover, which has a bearing on future damages in a legal matter; to make prospective decisions about a patient's medical treatment, which might raise legal issues in insurance law and tort law, and ethical issues in relation to access to care; and to determine employment prospects and fitness on the basis of an individual's neurological risk profile (as discussed below). In light of these different applications, the development of use-specific privacy protections and legal and scientific standards is important.

Some uses of brain imaging data raise ethical questions $^{12-14}$ (BOX 6). One foreseeable use by employers and insurers might be to decline employment or insurance cover for individuals who are particularly pain-sensitive or who exhibit a predisposition to chronic pain. In the military and other high-risk professions, for example, entry into service and opportunities for advancement could involve a neurological fitness examination that assesses, among other factors, acute pain sensitivity, emotional reactivity and predispositions to chronic pain and post-traumatic stress disorder. Brain imaging data could also be used to advise patients of their personal risks and benefits of a surgical procedure, including the risks of chronic post-surgical pain, and to protect physicians from liability if such pain develops. These possible uses, and others that are not currently foreseeable, raise legal issues of neurological and medical privacy, and of equality of access to employment, health insurance, and medical care. The scanning process itself also raises legal issues, including licensure and training of personnel to perform the scans and interpret the findings, on the basis of shared standards that ensure rigour and permit scans to be cross-comparable.

\section{Neuroethics and the law}

Marginalization of subjective experience. The most meaningful gauge of a person's pain is their self-report. Neither the absence of a known cause nor an aberrant response to a stimulus negates the experience of pain. If a patient honestly reports pain, they have pain - a principle that is encapsulated in the IASP definition. This principle is a central tenet of pain management, and is important for achieving adequate pain control, particularly in some populations with chronic pain that remain frequently marginalized and discredited. Within legal systems, the use of measurement and verification technologies most frequently becomes mandatory for marginalized groups. Nevertheless, people with chronic pain could voluntarily obtain brain scans if they have the financial means, so as to validate and explain their pain. If brain imaging is to be accepted as evidence in legal cases related to chronic pain, issues of access to brain imaging (for example, financial, geographical and availability issues), and what the choice made by an individual about whether to use or not use brain imaging implies about them, must be considered.

Resource allocation. All systems, including medical and legal systems, have finite resources. In pain medicine, the most pressing issues remain the undertreatment of pain, disparities in access to treatment, and the need to develop effective and safe treatment options; thus, clinical or legal use of expensive brain imaging techniques for detection of chronic pain might deflect resources away from more-effective treatment. Limitations on legal resources are also relevant to the interest of the legal community in tools to deal with claims related to chronic pain. Legal decision-makers should evaluate whether brain imaging would improve the efficiency of dealing with such cases, relative to lower-cost options, and determine the relative burden on the parties involved.

Explicit and implicit requirements of use. The introduction of a test for chronic pain could exert a legal gravitational pull: once a test is available, judges, jurors and other decision-makers might expect to see test results in all cases. In this context, the absence of this evidence could lead decision-makers to make informal adverse inferences about the party who declined the test. In this way, the availability of a test could make it de facto mandatory in practice, if not in law ${ }^{122}$. Submission of a brain scan could also become a precondition of any claim relating to chronic pain; although a claimant could not be forced to undergo testing, any claimant who refused would forfeit the right to proceed.

Whether refusal to undergo a scan should result in adverse inferences and legal consequences depends, in part, on the reliability of any brain imaging tests that are introduced. Unless the technology is highly accurate, adverse inferences should not be made about claimants who refuse to undergo the scan, and no legal requirement should be put in place. Any legal requirement to have a brain scan must also balance issues of autonomy, efficiency, and resource allocation.

Medical privacy and data security. Medical records are private, but legal records are not, and medical evidence used in legal matters generally becomes part of the public record. Brain-based evidence that enters the public record might be particularly sensitive because, like DNA evidence, it could be used to make other inferences about the individual. The risk is particularly high if the brain imaging evidence exchanged between parties and used in court consists of the underlying data files, which parties involved in the case, or others, could manipulate. Legal systems should, therefore, develop practices for the protection of neurological data, and should develop standards and practices that courts and private parties that are custodians of the data must follow to ensure data security. 


\section{Implications and recommendations}

In the past few decades, the use of brain imaging and other technologies has led to a paradigm shift in our understanding of acute and chronic pain disorders, and revealed that these disorders are, at least in part, mediated by the CNS. With suitable standardization and validation, several forms of brain imaging could provide objective biomarkers of key characteristics of chronic pain. Identification of brain-based markers of chronic pain might be possible with further technological advancement, large-scale data acquisition across diverse groups of individuals, and strict application of standards of evidence to the assessment of potential pain biomarkers. Furthermore, brain imaging promises to enable assessment of risk factors for chronic pain, and identification of the mechanisms that underlie transition to and maintenance of chronic pain. Brainbased tests could serve as an adjunct to self-report and behavioural evidence that corroborates, contradicts, or says nothing about an individual's claims about pain, distress and impairment. Such tests would be particularly valuable for nonverbal populations (children and adults who are disabled, very young or have dementia, for example), cases in which the evidence from verbal reports and behaviour is conflicting, and for personalized pain management.

As brain imaging measures become increasingly acceptable for directing personalized pain management in clinical contexts, the pressure to use them for medico-legal purposes will increase. Such use of these tools, however, would be inappropriate and unethical until they were sufficiently validated. Today, the necessary scientific knowledge - including the specificity and sensitivity of such tests - and validated protocols to enable use of brain imaging evidence in the legal system do not exist. Until they do, use of brain-based measurements that do not meet minimum standards would be detrimental to health care and legal systems, potentially harmful to patients and claimants, and legally inappropriate (and consequently unethical). In our view, current brain-based measures fall short of the requisite standards for legal proceedings, but we do encourage their use for understanding brain mechanisms that underlie pain, factors that lead to persistence of pain, and targets in the brain for safe and effective pain control.
1. Treede, R. D. et al. A classification of chronic pain for ICD-11. Pain 156, 1003-1007 (2015).

2. Von Korff, M. et al. United States national pain strategy for population research: concepts, definitions, and pilot data. J. Pain 17, 1068-1080 (2016).

3. Haxby, J. V., Connolly, A. C. \& Guntupalli, J. S. Decoding neural representational spaces using multivariate pattern analysis. Annu. Rev. Neurosci. 37, 435-456 (2014)

4. Tong, F. \& Pratte, M. S. Decoding patterns of human brain activity. Annu. Rev. Psychol. 63, 483-509 (2012).

5. Institute of Medicine, Board on Health Sciences Policy, ¿ Committee on Advancing Pain Research, Care, and Education. Relieving pain in America: a blueprint for transforming prevention, care, education, and research. (The National Academies Press, 2011).

6. Fayaz, A., Croft, P., Langford, R. M., Donaldson, L. J. \& Jones, G. T. Prevalence of chronic pain in the UK: a systematic review and meta-analysis of population studies. BMJ Open 6, e010364 (2016).

7. Rice, A. S., Smith, B. H. \& Blyth, F. M. Pain and the global burden of disease. Pain 157, 791-796 (2016).

8. International Association for the Study of Pain (IASP) IASP Taxonomy [online] https://www.iasp-pain.org/ Taxonomy (2017).

9. Merskey, H. et al. Pain terms: a list with definitions and notes on usage. Recommended by the IASP Subcommittee on Taxonomy. Pain 6, 249-252 (1979).

10. Reardon, S. Neuroscience in court: the painful truth Nature 518, 474-476 (2015)

11. Davis, K. Personal injury lawyers turn to neuroscience to back claims of chronic pain. $A B A$ J. http://www. abajournal.com/magazine/article/personal_injury lawyers turn to neuroscience to back claims of chronic pain (2016).

12. Davis, K. D. Legal and ethical issues of using brain imaging to diagnose pain. PAIN Reports 1 , e577 (2016).

13. Davis, K. D., Racine, E. \& Collett, B. Neuroethical issues related to the use of brain imaging: can we and should we use brain imaging as a biomarker to diagnose chronic pain? Pain 153, 1555-1559 (2012).

14. Seminowicz, D. et al. Panel 1: legal and neuroscientific perspectives on chronic pain. J. Health Care Law Policy 18, 207-235 (2015).

15. Haanpaa, M. et al. NeuPSIG guidelines on neuropathic pain assessment. Pain 152, 14-27 (2011).

16. Loeser, J. D. \& Treede, R. D. The Kyoto protocol of IASP basic pain terminology. Pain 137, 473-477 (2008).
17. Davis, K. D., Kucyi, A. \& Moayedi, M. The pain switch: an "ouch" detector. Pain 156, 2164-2166 (2015). This article highlights the unique quality of pain and its brain representation.

18. Ni, M. R., Warnaby, C., Rogers, R., Jbabdi, S. \& Tracey, I. Slow-wave activity saturation and thalamocortical isolation during propofol anesthesia in humans. Sci. Transl. Med. 5, 208ra1 48 (2013).

19. Schweinhardt, P. \& Bushnell, M. C. Pain imaging in health and disease - how far have we come? J. Clin. Invest. 120, 3788-3797 (2010).

20. Kwan, C. L., Diamant, N. E., Mikula, K. \& Davis, K. D. Characteristics of rectal perception are altered in irritable bowel syndrome. Pain 113, 160-171 (2005).

21. Bushnell, M. C., Ceko, M. \& Low, L. A. Cognitive and emotional control of pain and its disruption in chronic pain. Nat. Rev. Neurosci. 14, 502-511 (2013).

22. Borsook, D. $\&$ Kalso, E. Transforming pain medicine: adapting to science and society. Eur. J. Pain 17, 1109-1125 (2013).

23. Petrovic, P., Kalso, E., Petersson, K. M. \& Ingvar, M. Placebo and opioid analgesia - imaging a shared neuronal network. Science 295, 1737-1740 (2002).

24. Kucyi, A., Salomons, T. V. \& Davis, K. D. Mind wandering away from pain dynamically engages antinociceptive and default mode brain networks. Proc. Natl Acad. Sci. USA 110, 18692-18697 (2013).

25. Seminowicz, D. A. Believe in your placebo. J. Neurosci. 26, 4453-4454 (2006)

26. Tetreault, P. et al. Brain connectivity predicts placebo response across chronic pain clinical trials. PLoS Biol. 14, e1002570 (2016)

27. Borsook, D., Edwards, R., Elman, I., Becerra, L. \& Levine, J. Pain and analgesia: the value of salience circuits. Prog. Neurobiol. 104, 93-105 (2013).

28. Eippert, F. et al. Activation of the opioidergic descending pain control system underlies placebo analgesia. Neuron 63, 533-543 (2009).

29. Bantick, S. J. et al. Imaging how attention modulates pain in humans using functional MRI. Brain 125 310-319 (2002).

30. Erpelding, N. \& Davis, K. D. Neural underpinnings of behavioural strategies that prioritize either cognitive task performance or pain. Pain 154, 2060-2071 (2013).

31. Davis, K. D. \& Moayedi, M. Central mechanisms of pain revealed through functional and structural MRI. J. Neuroimmune Pharmacol. 8, 518-534 (2013). This article provides a summary of the functional and structural brain imaging approaches to studying acute and chronic pain, and the key findings from studies of acute pain and brain abnormalities in chronic pain.
32. Garcia-Larrea, L. \& Peyron, R. Pain matrices and neuropathic pain matrices: a review. Pain 154 (Suppl. 1), S29-S43 (2013).

This article presents an important summary of current concepts relating to the brain representation of pain.

33. Baliki, M. N. \& Apkarian, A. V. Nociception, pain, negative moods, and behavior selection. Neuron $\mathbf{8 7}$ 474-491 (2015)

34. Malfliet, A. et al. Brain changes associated with cognitive and emotional factors in chronic pain: a systematic review. Eur. J. Pain 21, 769-786 (2017).

35. Apkarian, A. V., Bushnell, M. C., Treede, R. D. \& Zubieta, J. K. Human brain mechanisms of pain perception and regulation in health and disease. Eur J. Pain 9, 463-484 (2005).

36. Morton, D. L., Sandhu, J. S. \& Jones, A. K. Brain imaging of pain: state of the art. J. Pain Res. 9, 613-624 (2016)

37. Mayer, E. A., Gupta, A., Kilpatrick, L. A. \& Hong, J. Y. Imaging brain mechanisms in chronic visceral pain Pain 156 (Suppl. 1), S50-S63 (2015).

38. Ploner, M., Sorg, C. \& Gross, J. Brain rhythms of pain. Trends Cogn. Sci. 21, 100-110 (2017).

39. lannetti, G. D., Hughes, N. P., Lee, M. C. \& Mouraux, A. Determinants of laser-evoked EEG responses: pain perception or stimulus saliency? J. Neurophysiol. 100, 815-828 (2008).

40. Clark, J. A., Brown, C. A., Jones, A. K. \& El-Deredy, W. Dissociating nociceptive modulation by the duration of pain anticipation from unpredictability in the timing of pain. Clin. Neurophysiol. 119, 2870-2878 (2008).

41. Mouraux, A., Diukova, A., Lee, M. C., Wise, R. G. \& lannetti, G. D. A multisensory investigation of the functional significance of the "pain matrix". Neuroimage 54, 2237-2249 (2011).

42. Wiech, K. et al. Anterolateral prefrontal cortex mediates the analgesic effect of expected and perceived control over pain. J. Neurosci. 26, 11501-11509 (2006).

43. Bingel, U. et al. The effect of treatment expectation on drug efficacy: imaging the analgesic benefit of the opioid remifentanil. Sci. Transl. Med. 3, 70ra14 (2011)

44. Melzack, R. Pain and the neuromatrix in the brain. J. Dent. Educ. 65, 1378-1382 (2001).

45. Melzack, R. From the gate to the neuromatrix. Pain (Suppl. 6), S121-S126 (1999).

46. Tracey, I. Nociceptive processing in the human brain Curr. Opin. Neurobiol. 15, 478-487 (2005).

47. Legrain, V., lannetti, G. D., Plaghki, L. \& Mouraux, A The pain matrix reloaded: a salience detection system for the body. Prog. Neurobiol. 93, 111-124 (2011). 
48. Iannetti, G. D. \& Mouraux, A. From the neuromatrix to the pain matrix (and back). Exp. Brain Res. 205, 1-12 (2010).

This review summarizes evidence that most brain activity related to pain is not specific to pain but rather represents the saliency content of the stimulus.

49. Hu, L. \& lannetti, G. D. Painful issues in pain prediction. Trends Neurosci. 39, 212-220 (2016).

50. Kucyi, A. \& Davis, K. D. The dynamic pain connectome. Trends Neurosci. 38, 86-95 (2015).

This article introduces the concept that pain is determined by both static and dynamic brain connectivity in ascending and descending pathways as well as the default mode and salience networks.

51. Rosa, M. J. \& Seymour, B. Decoding the matrix: benefits and limitations of applying machine learning algorithms to pain neuroimaging. Pain 155, 864-867 (2014).

52. Borsook, D., Becerra, L. \& Hargreaves, R. Biomarkers for chronic pain and analgesia. Part 2: how, where, and what to look for using functional imaging. Discov. Med. 11, 209-219 (2011).

53. Borsook, D., Becerra, L. \& Hargreaves, R. Biomarkers for chronic pain and analgesia. Part 1: the need, reality, challenges, and solutions. Discov. Med. 11 , 197-207 (2011).

54. Woo, C. W., Chang, L. J., Lindquist, M. A. \& Wager, T. D. Building better biomarkers: brain models in translational neuroimaging. Nat. Neurosci. 20, 365-377 (2017).

55. Labus, J. S. et al. Multivariate morphological brain signatures predict patients with chronic abdominal pain from healthy control subjects. Pain 156 1545-1554 (2015).

56. Ung, H. et al. Multivariate classification of structural MRI data detects chronic low back pain. Cereb. Cortex 24, 1037-1044 (2014). This study described how brain structure can be used to decode lower back pain.

57. Bagarinao, E. et al. Preliminary structural MRI based brain classification of chronic pelvic pain: a MAPP network study. Pain 155, 2502-2509 (2014).

58. Kuner, R. \& Flor, H. Structural plasticity, connectivity and reorganisation in chronic pain. Nat. Rev. Neurosci. 18, 20-30 (2016)

59. Poldrack, R. A. Inferring mental states from neuroimaging data: from reverse inference to largescale decoding. Neuron 72, 692-697 (2011).

60. Haynes, J. D. A. Primer on pattern-based approaches to fMRI: principles, pitfalls, and perspectives. Neuron 87, 257-270 (2015)

61. Wager, T. D. et al. An fMRI-based neurologic signature of physical pain. N. Engl. J. Med. 368, 1388-1397 (2013).

A seminal study showing that heat pain intensity in an individual can be predicted from fMRI signals.

62. Woo, C. W. \& Wager, T. D. Neuroimaging-based biomarker discovery and validation. Pain 156 , 1379-1381 (2015).

63. Lindquist, M. A et al. Group-regularized individual prediction: theory and application to pain. Neuroimage 145, 274-287 (2017).

64. Lopez-Sola, M. et al. Towards a neurophysiological signature for fibromyalgia. Pain 158, 34-47 (2017). This study shows decoding of a chronic pain condition from brain activity.

65. Woo, C. W. \& Wager, T. D. What reliability can and cannot tell us about pain report and pain neuroimaging Pain 157, 511-513 (2016)

66. Cecchi, G. A. et al. Predictive dynamics of human pain perception. PLoS Comput. Biol. 8, e1002719 (2012).

67. Brodersen, K. H. et al. Decoding the perception of pain from $\mathrm{fMRI}$ using multivariate pattern analysis. Neuroimage 63, 1162-1170 (2012).

68. Duff, E. P. et al. Learning to identify CNS drug action and efficacy using multistudy fMRI data. Sci Transl. Med. 7, $274 \mathrm{ra} 16$ (2015).

69. Schulz, E., Zherdin, A., Tiemann, L., Plant, C. $\delta$ Ploner, M. Decoding an individual's sensitivity to pain from the multivariate analysis of EEG data. Cereb. Cortex 22, 1118-1123 (2012) This study represents the first cross-subject prediction of pain from EEG activity.

70. Brown, J. E., Chatterjee, N., Younger, J. \& Mackey, S. Towards a physiology-based measure of pain: patterns of human brain activity distinguish painful from nonpainful thermal stimulation. PLOS ONE 6, e24124 (2011).

71. Liang, M., Mouraux, A., Hu, L. \& lannetti, G. D. Primary sensory cortices contain distinguishable spatial patterns of activity for each sense. Nat. Commun. 4, 1979 (2013).

72. Iannetti, G. D., Salomons, T. V., Moayedi, M., Mouraux, A. \& Davis, K. D. Beyond metaphor: contrasting mechanisms of social and physical pain. Trends Cogn. Sci. 17, 371-378 (2013). This study highlights the overlap of pain and other dimensions, such as salience, that leads to the problem of reverse inference in interpreting pain imaging data.

73. Todd, M. T., Nystrom, L. E. \& Cohen, J. D. Confounds in multivariate pattern analysis: theory and rule representation case study. Neuroimage 77, 157-165 (2013).

74. Chang, L. J., Gianaros, P. J., Manuck, S. B., Krishnan, A. \& Wager, T. D. A. Sensitive and specific neural signature for picture-induced negative affect. PLoS Biol. 13, e1002180 (2015)

75. Krishnan, A. et al. Somatic and vicarious pain are represented by dissociable multivariate brain patterns. Elife. 5, e15166 (2016)

76. Woo, C. W. et al. Separate neural representations for physical pain and social rejection. Nat. Commun. $\mathbf{5}$, 5380 (2014)

77. Becker, S., Gandhi, W., Pomares, F., Wager, T. D. \& Schweinhardt, P. Orbitofrontal cortex mediates pain inhibition by monetary reward. Soc. Cogn. Affect. Neurosci. 12, 651-661 (2017)

78. Brascher, A. K., Becker, S., Hoeppli, M. E. \& Schweinhardt, P. Different brain circuitries mediating controllable and uncontrollable pain. J. Neurosci. 36 5013-5025 (2016).

79. Woo, C. W., Roy, M., Buhle, J. T. \& Wager, T. D. Distinct brain systems mediate the effects of nociceptive input and self-regulation on pain. PLOS Biol. 13, e 1002036 (2015)

80. Woo, C. W. et al. Quantifying cerebral contributions to pain beyond nociception. Nat. Commun. 8, 14211 (2017).

81. Tracey, I. Neuroimaging mechanisms in pain: from discovery to translation. Pain 158 (Suppl. 1) S115-S122 (2017)

82. Seminowicz, D. A. ¿ Davis, K. D. Interactions of pain intensity and cognitive load: the brain stays on task. Cereb. Cortex 17, 1412-1422 (2007).

83. Lee, M. C., Zambreanu, L., Menon, D. K. \& Tracey, I. Identifying brain activity specifically related to the maintenance and perceptual consequence of central sensitization in humans. J. Neurosci. 28 11642-11649 (2008)

84. Schweinhardt, P. et al. An fMRI study of cerebral processing of brush-evoked allodynia in neuropathic pain patients. Neuroimage 32, 256-265 (2006)

85. Gwilym, S. E. et al. Psychophysical and functional imaging evidence supporting the presence of central sensitization in a cohort of osteoarthritis patients. Arthritis Rheum. 61, 1226-1234 (2009).

86. Mailis-Gagnon, A. et al. Altered central somatosensory processing in chronic pain patients with "hysterical" anesthesia. Neurology 60 1501-1507 (2003)

87. Koyama, T., McHaffie, J. G., Laurienti, P. J. \& Coghill, R. C. The single-epoch fMRI design: validation of a simplified paradigm for the collection of subjective ratings. Neuroimage 19, 976-987 (2003)

88. Coghill, R. C., Sang, C. N., Maisog, J. M. \& ladarola, M. J. Pain intensity processing within the human brain: a bilateral, distributed mechanism. J. Neurophysiol. 82, 1934-1943 (1999).

89. Kwan, C. L. et al. Abnormal forebrain activity in functional bowel disorder patients with chronic pain Neurology 65, 1268-1277 (2005)

90. Davis, K. D., Kwan, C. L., Crawley, A. P. \& Mikulis, D. J. Event-related fMRI of pain: entering a new era in imaging pain. Neuroreport 9, 3019-3023 (1998)

91. Atlas, L. Y., Lindquist, M. A., Bolger, N. \& Wager, T. D. Brain mediators of the effects of noxious heat on pain Pain 155, 1632-1648 (2014).

92. Davis, K. D., Pope, G. E., Crawley, A. P. \& Mikulis, D. J. Neural correlates of prickle sensation: a perceptrelated fMRI study. Nat. Neurosci. 5, 1121-1122 (2002).

93. Davis, K. D., Pope, G. E., Crawley, A. P. \& Mikulis, D. J. Perceptual illusion of "paradoxical heat" engages the insular cortex. J. Neurophysiol. 92, 1248-1251 (2004)

94. Apkarian, A. V., Krauss, B. R., Fredrickson, B. E. \& Szeverenyi, N. M. Imaging the pain of low back pain functional magnetic resonance imaging in combination with monitoring subjective pain perception allows the study of clinical pain states. Neurosci. Lett. 299, 57-60 (2001)

95. Baliki, M. N. et al. Chronic pain and the emotional brain: specific brain activity associated with spontaneous fluctuations of intensity of chronic back pain. J. Neurosci. 26, 12165-12173 (2006).

96. Porro, C. A., Lui, F., Facchin, P., Maieron, M. \& Baraldi, P. Percept-related activity in the human somatosensory system: functional magnetic resonance imaging studies. Magn. Reson. Imaging 22, 1539-1548 (2004)

97. Davis, K. D. Cold-induced pain and prickle in the glabrous and hairy skin. Pain 75, 47-57 (1998)

98. Davis, K. D. \& Pope, G. E. Noxious cold evokes multiple sensations with distinct time courses. Pain 98, 179-185 (2002).

99. Rogachov, A. et al. Regional brain signal variability: a novel indicator of pain sensitivity and coping. Pain 157, 2483-2492 (2016)

100. Mansour, A. et al. Global disruption of degree rank order: a hallmark of chronic pain. Sci. Rep. 6, 34853 (2016).

This study uses graph theory to demonstrate that chronic pain is associated with a global change in brain connectivity.

101. Apkarian, A. V., Baliki, M. N. \& Farmer, M. A. Predicting transition to chronic pain. Curr. Opin Neurol. 26, 360-367 (2013).

102. Baliki, M. N., Mansour, A. R., Baria, A. T. \& Apkarian, A. V. Functional reorganization of the default mode network across chronic pain conditions. PLOS ONE 9, e106133 (2014).

103. Cheng, J. C., Erpelding, N., Kucyi, A., DeSouza, D. D. \& Davis, K. D. Individual differences in temporal summation of pain reflect pronociceptive and antinociceptive brain structure and function. J. Neurosci. 35, 9689-9700 (2015)

104. Kucyi, A. \& Davis, K. D. The neural code for pain: from single-cell electrophysiology to the dynamic pain connectome. Neuroscientist 23, 397-414 (2017).

105. Tracey, I. \& Johns, E. The pain matrix: reloaded or reborn as we image tonic pain using arterial spin labelling. Pain 148, 359-360 (2010)

106. Loggia, M. L. et al. Default mode network connectivity encodes clinical pain: an arterial spin labeling study. Pain 154, 24-33 (2013).

107. Hemington, K. S., Wu, Q., Kucyi, A., Inman, R. D. \& Davis, K. D. Abnormal cross-network functional connectivity in chronic pain and its association with clinical symptoms. Brain Struct. Funct. 221 4203-4219 (2016).

108. Coulombe, M. A. et al. Lower functional connectivity of the periaqueductal gray is related to negative affect and clinical manifestations of fibromyalgia. Front. Neuroanat. http://dx.doi.org/10.3389/ fnana.2017.00047 (2017).

109. Kutch, J. J. et al. Resting-state functional connectivity predicts longitudinal pain symptom change in urologic chronic pelvic pain syndrome: a MAPP network study. Pain 158, 1069-1082 (2017).

110. Poldrack, R. A. Can cognitive processes be inferred from neuroimaging data? Trends Cogn. Sci. 10 59-63 (2006).

111. Wager, T. D. et al. Pain in the ACC? Proc. Natl Acad. Sci. USA 113, E2474-E2475 (2016).

112. Nichols, T. E. et al. Best practices in data analysis and sharing in neuroimaging using MRI. Nat. Neurosci. 20, 299-303 (2017).

113. Logothetis, N. K. Intracortical recordings and fMRI: an attempt to study operational modules and networks simultaneously. Neuroimage 62, 962-969 (2012).

114. Sobczyk, O. et al. Assessing cerebrovascular reactivity abnormality by comparison to a reference atlas. J. Cereb. Blood Flow Metab. 35, 213-220 (2015).

115. Pillai, J. J. \& Mikulis, D. J. Cerebrovascular reactivity mapping: an evolving standard for clinical functional imaging. AJNR Am. J. Neuroradiol. 36, 7-13 (2015)

116. Para, A. E. et al. Invalidation of fMRI experiments secondary to neurovascular uncoupling in patients with cerebrovascular disease. J. Magn. Reson. Imaging http://dx.doi.org/10.1002/jmri.25639 (2017).

117. Davis, K. D. Neurophysiological and anatomical considerations in functional imaging of pain. Pain 105, 1-3 (2003)

118. Pustilnik, A. C. A brain-based model of chronic pain can increase accuracy and fairness in law. Harvard Rev. Psychiatry http://dx.doi.org/10.1097/ HRP.0000000000000175 (2017). 
119. Mittenberg, W., Patton, C., Canyock, E. M. \& Condit, D. C. Base rates of malingering and symptom exaggeration. J. Clin. Exp. Neuropsychol. 24, 1094-1102 (2002).

120. McCabe, D. P. \& Castel, A. D. Seeing is believing the effect of brain images on judgments of scientific reasoning. Cognition 107, 343-352 (2008).

121. Denk, F., McMahon, S. B. \& Tracey, I. Pain vulnerability: a neurobiological perspective. Nat. Neurosci. 17, 192-200 (2014).

122. Cole, S. A. \& Dioso-Villa, R. Investigating the 'CSI Effect' effect: media and litigation crisis in criminal law. Stanford Law Rev. 61, 1335-1374 (2009).

123. National Research Council of the National Academies. Emerging Cognitive Neuroscience and Related Technologies (The National Academies Press, 2008).

124. The Royal Society. Brain waves. Royal Society Projects https://royalsociety.org/topics-policy/projects/ brain-waves/ (2011).

125. Mur, M., Bandettini, P. A. \& Kriegeskorte, N. Revealing representational content with patterninformation $\mathrm{fMRI}$ - an introductory guide. Soc. Cogn. Affect. Neurosci. 4, 101-109 (2009).
126. Huang, G. et al A novel approach to predict subjective pain perception from single-trial laserevoked potentials. Neuroimage 81, 283-293 (2013).

\section{Acknowledgements}

The task force thanks the International Association for the Study of Pain (IASP) past president, Dr Rolf-Detlef Treede, for recognizing the importance of the issue addressed in this Consensus Statement and supporting the creation of this presidential task force during his term as the IASP President. We also thank current IASP President Dr Judith Turner for her support of this task force.

\section{Author contributions}

All authors researched data for the article, made substantial contributions to discussions of the content, wrote components of the article, and reviewed and/or edited the manuscript before submission.

\section{Competing interests statement}

The authors declare that they have no competing interests.

Publisher's note

Springer Nature remains neutral with regard to jurisdictional claims in published maps and institutional affiliations.

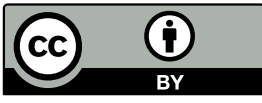

This work is licensed under a Creative Commons Attribution 4.0 International License. The images or other third party material in this

article are included in the article's Creative Commons license, unless indicated otherwise in the credit line; if the material is not included under the Creative Commons license, users will need to obtain permission from the license holder to reproduce the material. To view a copy of this license, visit http://creativecommons.org/licenses/by/4.0/.

\section{FURTHER INFORMATION}

US Centers for Disease Control and Prevention Office of Public Health Genomics ACCE (analytic validity, clinical validity, clinical utility and associated ethical, legal and social implications) Model Project:

http://www.cdc.gov/genomics/gtesting/acce/index.htm

The US BRAIN (Brain Research through Advancing

Innovative Neurotechnologies) Initiative:

https://www.braininitiative.nih.gov/

The European Union Human Brain Project:

https://www.humanbrainproject.eu/

The Japanese Brain/MINDS Project: http://brainminds.jp/en/ 